\title{
EFECTO HIPOTENSOR DESPUÉS DE EJERCICIO DE RESISTENCIA EN MUJERES MAYORES CON HIPERTENSIÓN
}

\section{HYPOTENSIVE EFFECT AFTER RESISTANCE EXERCISE IN OLDER WOMEN WITH HYPERTENSION}

De Oliveira Marco Aurélio Gomes ${ }^{1}$; Borba-Pinheiro Cláudio Joaquim ${ }^{1,2,3}$; da Rocha-Junior Olavo Raimundo Macêdo ${ }^{1}$;

1. Universidade do Estado do Pará (UEPA) Campus XIII. Laboratório de Treinamento Resistido e Saúde (LERES), Tucuruí - Pará, Brasil

2. Instituto Federal do Pará (IFPA). Grupo de Pesquisa em Saúde e Saneamento, Tucuruí-Pará, Brasil.

3. Universidade Federal do Estado do Rio de Janeiro (UNIRIO). Programa de Pós graduação Stricto Sensu (PPGEnfBio). Laboratório de Bio ciências da Motricidade Humana (LABIMH), Rio de Janeiro, Brasil.

DE OLIVEIRA M.A.G.; BORBA-PINHEIRO C.J.; DA ROCHA-JUNIOR O.R.M.; REIS THAÍSA É.T.; MONTEIRO-SANTOS R.; SOUZA A.C. \& WALSH-MONTEIRO A. Efecto hipotensor después de ejercicio de resistencia en mujeres mayores con hipertensión. Mot. Hum. 13(1):36-44; 2012.

\section{RESUMEN}

El objetivo fue evaluar los efectos de dos programas de 10 sesiones de entrenamiento de resistencia sobre la hipotensión sistémica de mujeres mayores hipertensas. 15 mujeres hipertensos distribuidos aleatoriamente en dos grupos: dos sesiones de entrenamiento / semana (GER 2xSm, $n=7$ a 62,3 8 8,6 años), y tres sesiones de entrenamiento / semana (GER 3xSm, $n=8$, con 57,7 \pm 7,9 años) fueron sometidos a un mismo programa de entrenamiento de resistencia en días alternos con el $70 \%$ de la carga máxima de 10 repeticiones (10RM) en ejercicios durante 10 sesiones. Se utilizó ANOVA para medidas repetidas en el análisis de la estadística. Se encontraron diferencias significativas en la comparación dentro del grupo, tanto para el GER 3xSm (PAS; $\Delta \%=-5,6 \%, p=0,001$ y la PAD $\Delta \%=-4,5 \%, p=$ $0,002)$ y de GER 2xSm (PAS; ( $\Delta \%=-6,2 \%, p=0,01$ y la PAD $\Delta \%=-6,1 \%, p=0,03)$ sin embargo, no hubo diferencias $(p<0,05)$ en la comparación entre los grupos. Se concluye que el entrenamiento de resistencia hecho dos veces y tres veces por semana con ejercicios de intensidad moderada proporcionó un efecto hipotensor para PAS y PAD en las mujeres mayores hipertensas.

Palabras Clave: Hipotensión post-ejercicio, Hipertensión, Ejercicio de resistencia, Servicios de salud para ancianos.

\begin{abstract}
To evaluate the effects of two programs of 10 sessions of resistance training on systemic hypotension in hypertensive older women. 15 hypertensive older women separated into two groups: two training sessions / week (RTG $2 x$ week, $n=7$ to $62.3 \pm 8.6$ years old), and three training sessions / week (RTG $3 x$ week, $n=8$, with $57.7 \pm 7.9$ years) underwent the same resistance training program on alternate days with an intensity of $70 \%$ of 10 repetition maximum (10RM) for exercise during 10 sessions. ANOVA repeated measures test for statistical analysis was used. Significant differences were observed for intra-group comparison, both for the RTG 3xweek $(S B P ; \Delta \%=-5.6 \%, p=0.001$ and $D B P ; \Delta \%=-4.5 \%, p=0.002)$ and for RTG 2xweek (SBP; $(\Delta \%=-6.2 \%, p=0.01$ and DBP; $\Delta \%=-6.1 \% p=0.03)$. However, no differences were observed $(p<0,05)$ for comparison between groups. We conclude that resistance training performed two and three times per week with moderate intensity of effort provided a hypotensive effect for SBP and DBP in hypertensive older women.
\end{abstract}

Key words: post-exercise hypotension, hypertension, Resistance exercise, health services for the elderly. 
DE OLIVEIRA M.A.G.; BORBA-PINHEIRO C.J.; DA ROCHA-JUNIOR O.R.M.; REIS THAÍSA É.T.; MONTEIRO-SANTOS R.; SOUZA A.C. \& WALSH-MONTEIRO A. Efecto hipotensor después de ejercicio de resistencia en mujeres mayores con hipertensión. Mot. Hum. 13(1):36-44; 2012

\section{INTRODUCCIÓN}

El judo de alto rendimiento es una modalidad que requiLa hipertensión arterial (HA) tiene una proporción de uno caso en cuatro personas de la población vieja y en edad productiva por toda la América Latina, que se considera una enfermedad silenciosa cuyas consecuencias principales son la isquemia y las enfermedades cerebrovasculares, por lo que tiene un fuerte impacto económico (1). Sin embargo, se demostró que se puede prevenir mediante tratamiento oportuno y control de los factores de riesgo (1).

El American College of Sport Medicine (ACSM) recomienda la actividad física como una ayuda no farmacológica para el tratamiento y / o control de la hipertensión en la atención primaria de salud, con un mínimo de tres veces a la semana con un tiempo de 30 minutos (2).

La Organización Mundial de la Salud (OMS), complementa, lo que sugiere a las personas de 18-64 años la realización de ejercicios de resistencia para los grandes grupos musculares, con la frecuencia mínima de dos veces por semana (3).

La práctica de ejercicio tiende a reducir los niveles de presión en la presión arterial sistémica (PA), este fenómeno se denomina hipotensión post-ejercicio (HPE) y esto es considerado un excelente comportamiento fisiológico de control de presión, especialmente para las personas con hipertensión arterial (4). Así, cuanto mayor sea la magnitud y duración de la HPE, el efecto será más eficaz como una ayuda no farmacológico para reducción de la PA en reposo (5).

Por lo tanto, el ejercicio de resistencia (ER) como método auxiliar de la prevención y control de la hipertensión puede reducir al mínimo el riesgo de accidente cerebro vascular (ACV), debido a la reducción de $3 \mathrm{~mm} \mathrm{Hg}$ en la PA puede disminuir 5 - 9\% la morbilidad cardiovascular y entre los $8-14 \%$ de riesgo de ACV (6).

Teniendo en cuenta los beneficios que la realización de ER proporciona sobre la presión arterial por el fenómeno de la HPE, el estudio tiene como objetivo evaluar los efectos de dos programas de 10 sesiones de ER sobre la hipotensión sistémica en mujeres mayores hipertensas.

\section{MATERIAL Y MÉTODOS}

\section{Los participantes}

Los participantes del experimento fueron las mujeres sedentarias, de 50 años o más, inscritos en el proyecto de investigación y extensión institucionalizada en la Universidad de Pará (UEPA) en la localidad de Tucuruí-PA, Brasil. 15 mujeres con hipertensión controlada, fueron seleccionados y separadas de manera aleatoria en dos grupos, a saber: dos sesiones de entrenamiento a la semana (GER $2 \mathrm{xSm}, \mathrm{n}=7$ a $62,3 \pm 8,6$ años) y tres sesiones de entrenamiento a la semana (GER 3xSm, $\mathrm{n}=8$; con 57,7 \pm 7,9 años) la selección se muestra en la Figura 1.

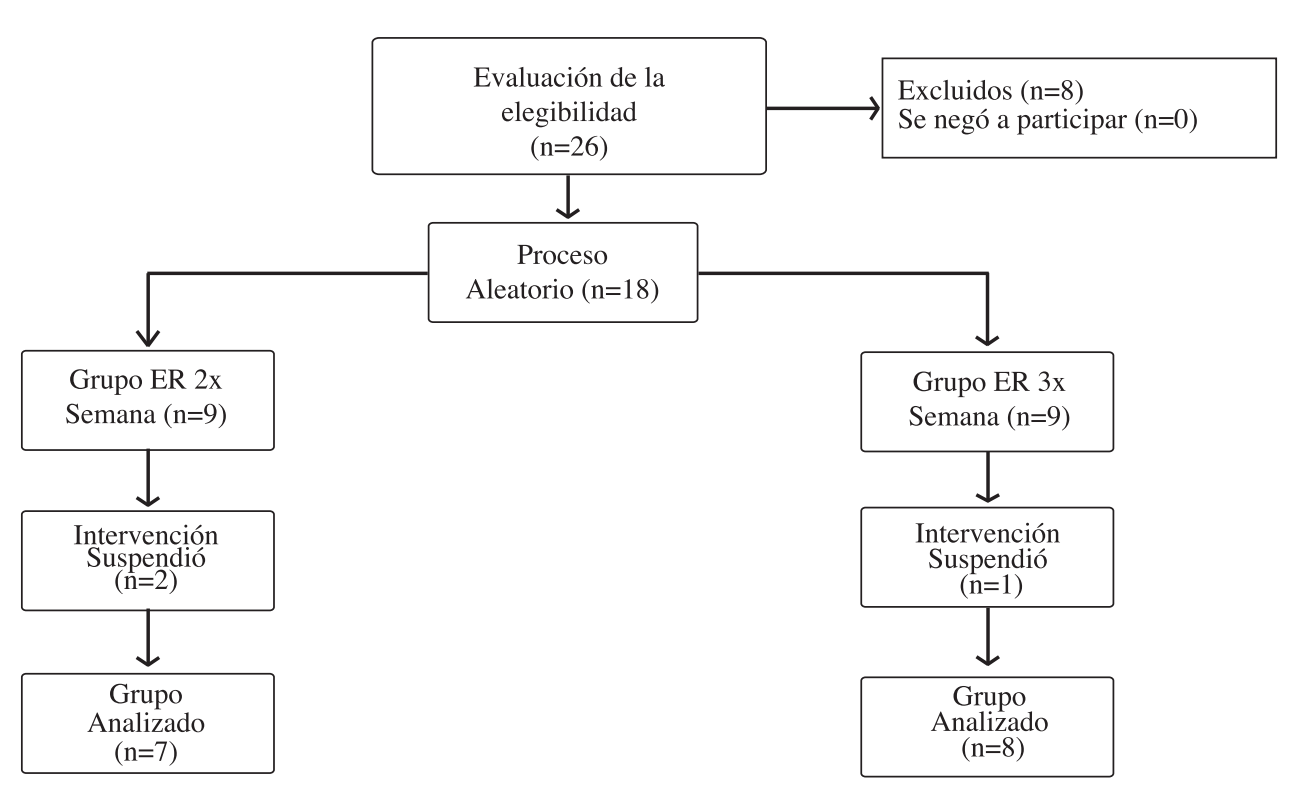

Figura 1: Presenta el proceso de selección de la muestra. 
DE OLIVEIRA M.A.G.; BORBA-PINHEIRO C.J.; DA ROCHA-JUNIOR O.R.M.; REIS THAÍSA É.T.; MONTEIRO-SANTOS R.; SOUZA A.C. \& WALSH-MONTEIRO A. Efecto hipotensor después de ejercicio de resistencia en mujeres mayores con hipertensión. Mot. Hum. 13(1):36-44; 2012

Los criterios de exclusión para la selección fueron los siguientes: crónicas limitaciones músculo-esqueléticos, enfermedades metabólicas, enfermedades del riñón, los usuarios de tabaco, alcohol y/o drogas ergogénicas, arritmias ventriculares inducido por el esfuerzo observadas por una prueba ergométrica anterior. Se incluyeron en el estudio, los voluntarios con 50 años de edad o más, con hipertensión, con tratamiento farmacológico y sin realizar ejercicio regular durante al menos seis meses. Los voluntarios recibieron instrucciones de no realizar otro tipo de ejercicio físico durante el período de estudio y mantener un horario de rutina de sueño y nutrición adecuada. Después de la orientación necesaria sobre el propósito y los procedimientos para los voluntarios, los participantes firmaron un formulario de consentimiento, en conformidad con la Resolución 196/96 del Consejo Nacional de Salud de Brasil que establece los criterios para la investigación ética con los seres humanos (7) y el proyecto fue aprobado por el comité de investigación de la Universidad UEPA.

\section{PROTOCOLOS}

\section{Evaluación de la composición corporal}

Inicialmente, se llevó a cabo una evaluación de las características del grupo de la muestra en peso y la altura utilizando una balanza antropométrica R-110, Welmy (B) (Brasil) acoplada con un estadiómetro. Para calcular el Índice de Masa Corporal (IMC) se utilizó la fórmula $\mathrm{IMC}=$ [peso $(\mathrm{kg}) /$ altura $\left.(\mathrm{cm})^{2}\right]$. Además, se obtuvo la cintura y la cadera medidas para determinar el índice de relación cintura-cadera (IRCC) por la fórmula IRCC= (circunferencia de la cintura $(\mathrm{cm}) /$ circunferencia de la cintura (cm) (8). Todas las mediciones seguido el establecido por el International Standards for Anthropometric Assessment (9).

\section{Evaluación de la Presión Arterial Sistémica}

La medición de la PA se realizó mediante la auscultación (ESFHS20M, G-Tech ®, Brasil) con los voluntarios sentados, el brazo izquierdo a la altura de los hombros sobre una superficie plana con la punta del manguito posicionado $2,5 \mathrm{~cm}$ por encima de la fosa cubital. La evacuación ocurrió $10 \mathrm{mmHg}$ por segundo, y se utilizó la $1^{\text {a }}$ y 5 a sonidos de Korotkoff para la distinción de la presión sistólica y diastólica de acuerdo con las recomendaciones de Petrie et al. (10). Los participantes se mantuvo en reposo en sillas cómodas por 10min para medición de la PA pre-entrenamiento y post-entrenamiento (11).

\section{Tratamiento Farmacológico}

Los sujetos del estudio se utilizaron los siguientes medicamentos durante el periodo de formación: inhibidores de la enzima convertidor de angiotensina (ECA) $(n=4)$; bloqueadores de los canales de $\mathrm{Ca}+2$ y inhibidores de la ECA $(n=5)$, diuréticos y de inhibidores de la ECA $(n=$ 6). Los fármacos fueron utilizados en forma de tableta de acuerdo con la prescripción del médico.

\section{Prueba de Fuerza Muscular}

En principio, se realizó una sesión de estiramiento en la preparación para la acción de los principales grupos musculares. Se llevó a cabo tres a cuatro series de ejercicios con cargas ligeras para la familiarización de los movimientos. Posteriormente, se practicó un máximo de cuatro intentos para llevar a cabo la prueba de 10RM, los intervalos de descanso entre los intentos deben ser de tres a cinco minutos, esta prueba se recomienda para las personas mayores y los no entrenados (12).

\section{Procedimientos para la intervención.}

Los participantes se sometieron a 10 sesiones de ER, donde un grupo practicó dos sesiones de entrenamiento / semana y el otro grupo practicó tres sesiones de entrenamiento / semana. El intervalo de recuperación fue de 48 horas entre cada sesión semanal, con un minuto de descanso entre series y entre ejercicios. El entrenamiento se llevó a cabo por la mañana con el inicio de las 07:00. Los siguientes ejercicios: flexión y extensión en la prensa de piernas $45^{\circ}$ y en la prensa de piernas horizontal, extensión de la rodilla, aducción de la cadera, flexión plantar, aducción del hombro (dorsal latísimo), flexión del codo, extensión del codo y aducción del hombro medial se realizaron en equipos de marca Physicus ${ }^{\circledR}$ (Brasil), con anillos de dos , cinco, 10,15 y $20 \mathrm{~kg}$ y pesos de dos a seis $\mathrm{kg}$ con tres series de 15 a 20 repeticiones y la intensidad de 70\% 10RM (12).

Los voluntarios pasaron por un período de adaptación de una semana para aprender la biomecánica del movimiento, la coordinación de la respiración (sin maniobra de valsalva), la velocidad de ejecución y la secuencia de los movimientos de los ejercicios. A continuación, los estiramientos se realizaron por el método estático (10s) para los grupos de músculos y luego se llevó a cabo la sesión de ER por el método de alternar segmentos. Todo el proceso de planificación y ejecución de ER fue realizado siguiendo el $\operatorname{ACSM}(2,12)$.

\section{Análisis Estadístico}

El análisis estadístico se realizó en el PASW ${ }^{\circledR} 17.0$ para Windows aceptando el valor de $\mathrm{p}<0,05$ con un error $(\alpha)=5 \%$. En principio, un análisis descriptivo se realizó con media y desviación estándar de la prueba previa de datos. Se llevó a cabo la análisis de normalidad con la prueba de Shapiro-Wilk y prueba posterior de Levene's con valor de $\mathrm{p}>0,05$; lo que permitió el uso de ANOVA para 
DE OLIVEIRA M.A.G.; BORBA-PINHEIRO C.J.; DA ROCHA-JUNIOR O.R.M.; REIS THAÍSA É.T.; MONTEIRO-SANTOS R.; SOUZA A.C. \& WALSH-MONTEIRO A. Efecto hipotensor después de ejercicio de resistencia en mujeres mayores con hipertensión. Mot. Hum. 13(1):36-44; 2012

medidas repetidas dentro e inter-grupos. Además, la potencia del experimento fue calculada con el error $(\beta)=20 \%$. Y, por último para calcular la diferencia porcentual fue elegido la fórmula de $\Delta \%=[($ Post-test - Test $) * 100 /$ Test $]$.

\section{RESULTADOS}

La Tabla 1 presenta los datos iniciales de las variables de los grupos estudiados.

\begin{tabular}{|c|c|c|c|c|}
\hline \multirow[b]{2}{*}{ Variables } & \multicolumn{2}{|c|}{ GER $3 \times$ Semana; $n=8$} & \multicolumn{2}{|c|}{ GER $2 \times$ Semana; n=7 } \\
\hline & Media & $\mathrm{DE}$ & Media & $\mathrm{DE}$ \\
\hline Edad (años) & 57,70 & 7,90 & 62,30 & 8,60 \\
\hline Masa (kg) & 70,10 & 15,30 & 64,10 & 6,60 \\
\hline Estatura (cm) & 151,20 & 0,05 & 151,10 & 0,04 \\
\hline IMC (kg/m²) & 30,30 & 5,20 & 27,90 & 1,70 \\
\hline $\operatorname{IRCC}(\mathrm{cm} / \mathrm{cm})$ & 0,870 & 0,81 & 0,91 & 0,06 \\
\hline PAS (mmHg) & 130,0 & 14,10 & 133,10 & 13,07 \\
\hline PAD (mmHg) & 79,30 & 4,90 & 80,70 & 3,40 \\
\hline \multicolumn{5}{|c|}{$\begin{array}{l}\text { DE= Desviación Estándar; IMC= Índice de masa corporal; IRCC=Índice Riesgo Cintura-Cadera; PAS=Presión Arterial Sistólica; } \\
\text { PAD=Presión Arterial Diastólica. }\end{array}$} \\
\hline
\end{tabular}

La Figura 2 muestra los valores medios de $\Delta \%$ entre las mediciones de la presión arterial sistólica (PAS) en el curso de 10 sesiones de GER 3 xsemana, donde $\Delta \%$ sufrió un efecto hipotensor: sesión $1 \Delta \%=-7,8 \%$; sesión $2 \Delta \%$ $=-4,6 \%$; sesión $3 \Delta \%=-3,05 \%$; sesión $4 \Delta \%=-1,9 \%$; sesión $5 \Delta \%=-8,7 \%$; sesión $6 \Delta \%=-4,1 \%$; sesión $7 \Delta \%$ $=-7,7 \%$; sesión $8 \Delta \%=-5,2 \%$; sesión $9 \Delta \%=-7,7 \%$ y sesión $10 \Delta \%=-5,05 \%$. Para la hipotensión sistólica del
GER 2xSemana, el resultado fue el siguiente: sesión 1 $\Delta \%=3,9 \%$; sesión $2 \Delta \%=-9,3 \%$; sesión $3 \Delta \%=-5,8 \%$; sesión $4 \Delta \%=-8,1 \%$; sesión $5 \Delta \%=4,4 \%$; sesión $6 \Delta \%$ $=-1,8 \%$; sesión $7 \Delta \%=-5,4 \%$; sesión $8 \Delta \%=-3,2 \%$; sesión $9 \Delta \%=-12,3 \% \%$ y sesión $10 \Delta \%=-8,2 \%$, mostrando una mejoría estadísticamente significativa dentro del grupo $\mathrm{p}<0,05$ tanto para GER 2xSemana como para el GER 3xSemana.

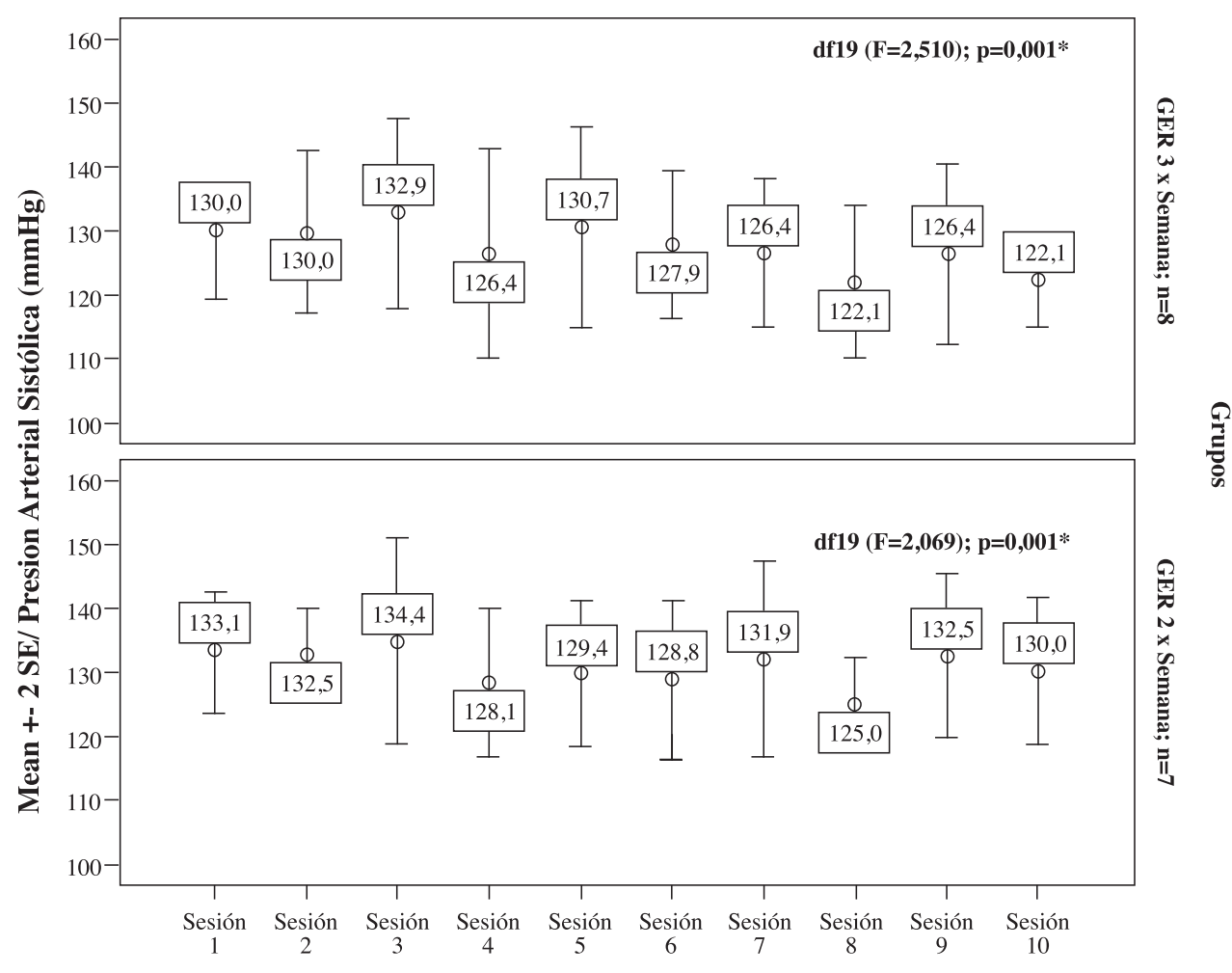

Figura 2: Análisis dentro de grupos de PAS. El símbolo (*) indica p $<0,05$ por ANOVA. 
En la Figura 3, que presenta los datos de la presión arterial diastólica (PAD) en la misma forma que la figura anterior, muestra que también hubo una mejoría estadísticamente significativa $\mathrm{p}<0,05$ dentro de ambos grupos de ER. Los valores delta $\%$ son los siguientes: sesión $1 \Delta \%$ $=-4,1 \%$; sesión $2 \Delta \%=-4,7 \%$, sesión $3 \Delta \%=-0,2 \%$; sesión $4 \Delta \%=-3,2 \%$, sesión $5 \Delta \%=-1,1 \%$, sesión $6 \Delta \%$ $=-5,6 \%$, sesión $7 \Delta \%=-11,6 \%$; sesión $8 \Delta \%=-10,4 \%$, sesión $9 \Delta \%=1,2 \%$ y sesión $10 \Delta \%=-1,3 \%$ para GER 3xSemana. Para la hipotensión diastólica del GER 2xSemana el resultado fue: sesión $1 \Delta \%=-4,9 \%$; sesión 2 $\Delta \%=-1,8 \%$; sesión $3 \Delta \%=-6,9 \%$; sesión $4 \Delta \%=-4,9 \%$; sesión $5 \Delta \%=0 \%$; sesión $6 \Delta \%=-3,06 \%$; sesión $7 \Delta \%=$ $0,4 \%$; sesión $8 \Delta \%=-1,8 \%$; sesión $9 \Delta \%=-8,9 \%$ y sesión $10 \Delta \%=-3,5 \%$.

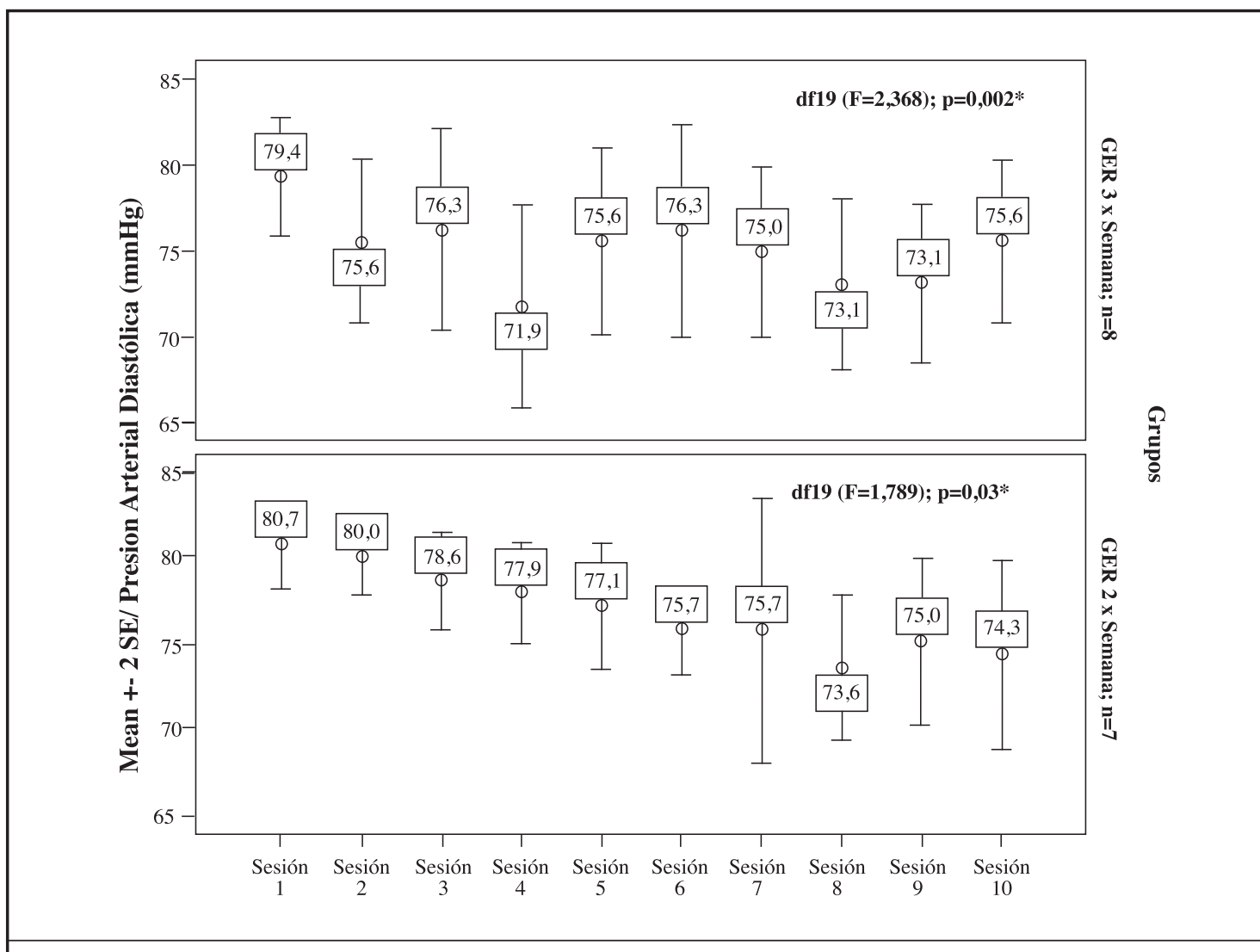

Figura 3: Análisis dentro de grupos de PAD. El Símbolo (*) indica $\mathrm{p}<0,05$ por ANOVA

Sin embargo, en la análisis entre los grupos no hubo diferencia estadísticamente significativa $(\mathrm{p}<0,05)$, donde el GER 3xSemana tuvo un efecto hipotensor en el PAS $(\Delta \%$ $=-5,6 \%$ ) ya el GER $2 x$ Semana tuvo un efecto hipotensor de la PAS $(\Delta \%=-6,2 \%)$ durante las 10 sesiones del estudio. Además, no hubo diferencias estadísticamente significativas entre los grupos $(\mathrm{p}<0,05)$ para la PAD, donde el GER 3xSemana tuvo un efecto hipotensor de los $(\Delta \%$ $=-4,5 \%)$ y el GER $2 x$ Semana tuvo un efecto hipotensor de $(\Delta \%=-6,1 \%)$ en el período de entrenamiento estudiado. La Figura 4 muestra el resultado de la comparación entre grupos con los valores de potencia de las pruebas, donde la media indica la potencia del experimento de 0,726 . 


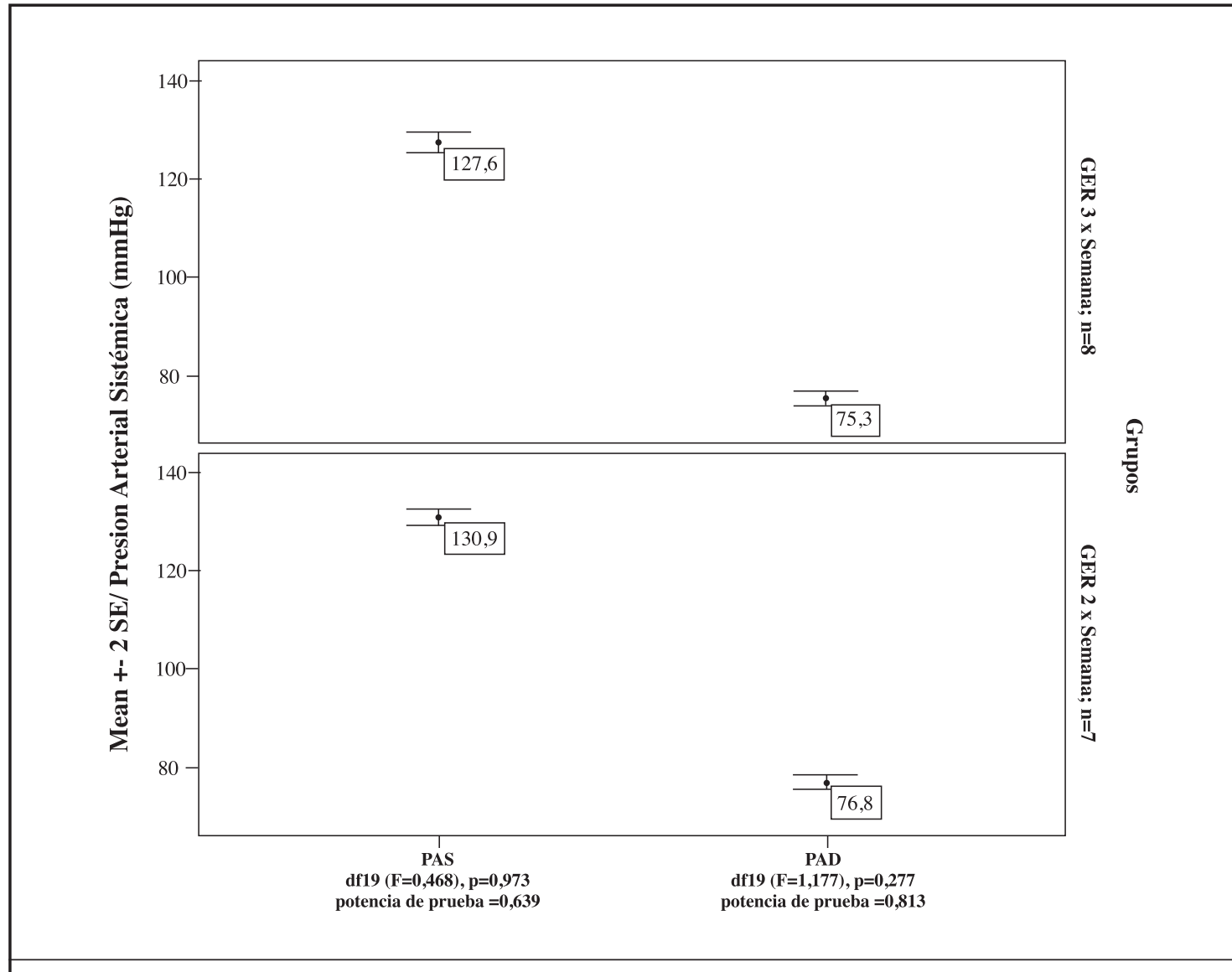

Figura 4: Análisis inter-grupos por la media de 10 mediciones de PAS e PAD por ANOVA.

\section{DISCUSIÓN}

La iniciación de una persona mayor en un programa de actividad física requiere más atención debido a la complejidad resultante de la senilidad, por ejemplo, el deterioro de su salud por la aparición de enfermedades pre-existentes y especialmente por la hipertensión. Sin embargo, la adherencia a la práctica contribuye directamente a la socialización, promoción de la salud y el bienestar de los individuos $(1,13)$.

Entre los principales problemas de salud en la vejez, la hipertensión merece destacarse por su alta prevalencia global y puede ser superior al $60 \%$ en la población de adultos mayores de América Latina $(1,14)$. La inactividad física es un importante factor de riesgo para la hipertensión, por lo que la OMS recomienda, en particular a los adultos mayores, la práctica regular de actividad física con la orientación profesional $(1,3)$. Además, la actividad física utilizado como instrumento auxiliar a la salud primaria y secundaria, podrían reducir los altos costos por las agencias gubernamentales de salud $(1,3)$.

El ER realizado por los voluntarios con un comportamiento sedentario en este estudio, mostró una reducción de la presión arterial, tanto sistólica como diastólica.

Costa et al. (15) también observaron reducciones significativas en la presión arterial en los ancianos hipertensos sedentarios a través del mismo ejercicio. Al comparar el efecto de la HPE en pacientes hipertensos sedentarios y entrenados, Costa et al. (15) se dieron cuenta de que el grupo sedentario mostró una mejor respuesta hipotensora después de ER.

El estudio Mediano et al. (16) en los individuos hipertensos de ambos sexos ( $n=20,61 \pm 12$ años $)$, que realizaron cuatro tipos diferentes de ejercicios (press de banca, prensa de piernas horizontal, remo y el tríceps en polea) presentaron como resultado una hipotensión entre uno 
DE OLIVEIRA M.A.G.; BORBA-PINHEIRO C.J.; DA ROCHA-JUNIOR O.R.M.; REIS THAÍSA É.T.; MONTEIRO-SANTOS R.; SOUZA A.C. \& WALSH-MONTEIRO A. Efecto hipotensor después de ejercicio de resistencia en mujeres mayores con hipertensión. Mot. Hum. 13(1):36-44; 2012

y tres series de 10RM. Sin embargo, la hipotensión fue mayor en el entrenamiento con tres series, lo que sugiere que un mayor volumen de ejercicio puede dar lugar a efectos a largo plazo de la hipotensión (16).

Por otro lado, el estudio de Simão y Santos (11) también con cuatro ejercicios (tracción dorsal en la polea, prensa de la pierna horizontal, flexión del codo y flexión de las rodillas.) con tres series de 10RM en individuos con normal presión arterial $(n=4$ mujeres, $21 \pm 0,8$ años y los hombres $\mathrm{n}=5,20,6 \pm 1,7$ años) no mostró resultados significativos para la PAS y PAD después del ejercicio.

Aunque, son pocos los estudios relacionados con la verificación de HPE a través de ER, las respuestas de hipotensión después del ejercicio parece ser más evidente en los pacientes hipertensos que en los normotensos $(2,17)$.

Después de la comparación de los dos grupos de ER, dos o tres veces a la semana, se observó ninguna diferencia significativa en la relación entre el número de sesiones semanales y la respuesta hipotensora. Este resultado indica que la práctica de dos sesiones semanales de ER es eficaz en la inducción de la HPE en mujeres mayores hipertensas.

Otro punto a destacar, aunque pocos estudios muestran reducciones de la PAD (18), hubo una mejora significativa en la PAD después del ER en este estudio (Figura 3) que confirma los estudios de Mediano et al. (16) y Moraes et al. (19).

En cuanto a la práctica de los ejercicios, la magnitud y duración de la HPE tienen una relación directa con la intensidad del ejercicio practicado, ya sea el ejercicio aeróbico o de resistencia. Boroujerdi et al. (20) demostró que el ejercicio de intensidad moderada, tal como se utiliza en este trabajo, aumentaron la magnitud de la HPE en comparación con el ejercicio de baja intensidad. Sin embargo, después del ejercicio las respuestas de hipotensión se encuentran diferenciadas en función del tipo de ejercicio realizado (21). Esto sugiere que son diferentes los eventos fisiológicos que regulan la HPE en los ejercicios aeróbicos y de resistencia (21).

Aunque no hay consenso sobre cuál sería el principal mecanismo fisiológico resultante en HPE, algunos estudios describen los elementos relacionados con este efecto. La reducción de la actividad autonómica simpática es una de las hipótesis identificadas como responsables de HPE (22). Floras et al. (23) notó una reducción en la actividad simpática asociada con HPE una hora después de una actividad aeróbica moderada. Para Halliwill (24) la reducción de la actividad simpática asociada con vasodilatadores conduce a una reducción en la resistencia vascular periférica (RVP) y, en consecuencia, disminución de la PA. Según Legramante et al. (25), la reducción de la RVP es un evento importante en la reducción de la PA, en consecuencia del efecto HPE.

El óxido nítrico y las prostaglandinas son mediadores con efectos vasodilatadores con el aumento de la liberación durante el ejercicio (26). Sin embargo, los estudios controlados que los niveles de estos mediadores, que bloquean la síntesis de óxido nítrico (27) o la secreción de prostaglandinas (28) identificaron la ocurrencia de la HPE, mismo sin influencia de las sustancias inhibidoras.

El efecto de las catecolaminas no parece tan crucial acerca de HPE. Los estudios muestran que los niveles de adrenalina y norepinefrina son similares a los obtenidos antes de hacer ejercicio en las personas que han expresado HPE (5).

El gasto cardíaco también es otra variable que no tiene un consenso sobre sus efectos en la HPE. Un estudio asocia con disminución del gasto cardíaco a HPE mediante la reducción del volumen de eyección del ventrículo y la frecuencia cardiaca en pacientes hipertensos (17). Sin embargo, otros estudios equivalentes con los pacientes hipertensos tenían HPE con el aumento del gasto cardíaco (29) o ningún cambio (25).

Por lo tanto, hay una gran cantidad de lagunas sobre el mecanismo fisiológico asociado con HPE que quedan por explorar y aclarar, sin embargo, muchos estudios han sugerido que los ejercicios que utilizan más la masa muscular, como las piernas, ya que pueden influir en la duración de la respuesta (30) y su magnitud (31) sobre la HPE. En este estudio, hemos tratado de hacer uso de todos los grupos musculares principales del cuerpo, no restringido a ciertos grupos de músculos.

Con relación entre la PAS y PAD, el GER 3xSm mostró una mayor reducción de la PAS $(\Delta \%=-5,6 \%)$ en comparación con el $\operatorname{PAD}(\Delta \%=-4,5 \%)$. Según algunos estudios, esta característica es probablemente debido a la reducción de la actividad simpática en el miocardio, lo que conduce a una reducción de la contractilidad del músculo y el gasto cardíaco $(5,17)$, y no la reducción de RVP. Esta característica tiende a estar asociada con el proceso de envejecimiento que se traduce en una menor producción y respuesta de sustancias vasodilatadores en los músculos arteriales (32). 
DE OLIVEIRA M.A.G.; BORBA-PINHEIRO C.J.; DA ROCHA-JUNIOR O.R.M.; REIS THAÍSA É.T.; MONTEIRO-SANTOS R.; SOUZA A.C. \& WALSH-MONTEIRO A. Efecto hipotensor después de ejercicio de resistencia en mujeres mayores con hipertensión. Mot. Hum. 13(1):36-44; 2012

En GER 2xSm, no hubo diferencia entre la PAS $(\Delta \%=$ $-6,2 \%)$ y $\operatorname{PAD}(\Delta \%=-6,1 \%)$. Si se considera que se encontró HPE en ambos grupos de ER (de dos a tres veces por semana), que la PAD mostró mejor reducción en el GER2xSm, y que no hubo diferencias en el efecto si se compara la HPE los dos grupos, suponemos que ER dos veces a la semana era suficiente para tener un efecto sobre la reducción de la presión arterial en voluntarios, en comparación con ER tres veces a la semana. Sin embargo, una pequeña muestra y el control de la dieta baja en sodio se considera las limitaciones de este estudio.

\section{CONCLUSIONES}

El ER con la intensidad moderado (70\%) en las mujeres mayores con hipertensión arterial ha demostrado ser beneficiosa en la inducción de $\mathrm{PEH}$ para entrenamiento dos o tres veces por semana. Sin embargo, el entrenamiento dos veces por semana parece ser suficiente para lograr la reducción de la presión arterial inducida por el ejercicio, puesto que la comparación entre los grupos no mostraron diferencias significativas.

\section{REFERENCIAS BIBLIOGRÁFICAS}

1. Organización Panamericana de Salud (OPS). $126^{\text {a }}$ reunión del Comité ejecutivo: relatoría final. Washington, D.C., junio, 2000.

2. ACMS - American College of Sports Medicine. Exercise and Hypertension. Medicine \& Science in Sports \& Exercise. 2004;36(3):533-553.

3. WHO - World Health Organization. Global recommendations on physical activity for health. WHO Library Cataloguing-in-Publication Data, 2010.

4. Polito PD; Farinatti PTV. Comportamiento de la presión arterial después de los ejercicios de contra-resistencia: una revisión sistemática sobre la determinación de las variables y los posibles mecanismos. Rev Bras Med Esporte. 2006;12(6):386-392.

5. MacDonald JR. Potential causes, mechanisms, and implications of post exercise hypotension. J Hum Hypertens. 2002;16:225-236.

6. Meka N; Katragadda S; Cherian B; Arora RR. Review. Endurance exercise and resistance training in cardiovascular disease. Ther Adv Cardiovasc Dis. 2008;2(2):115-121.

7. Brasil. Resolución 196/96 del Consejo Nacional de Salud de Brasil, 1996

8. Heyward VH. Evaluación física y prescripción de ejercicio. São Paulo: Artmed, 2002.

9. Marfell-Jones M; Olds T; Stewart A; Carter L. International standards for anthropometric assessment. ISAK: Potchefstroom, South Africa, 2006.

10. Petrie JC; O'brien ET; Littles WA; De Swiet M. British Hypertension Society: Recommendations on blood pressure measurement. Br Med J. 1986;293: 611-615.

11. Simão R; Santos EMR. (2005). Comportamiento de la presión arterial después de una sesión de ejercicios de resistencia. Fitness \& Performance Journal. 2005;4(4): 227 - 231.
12. ACMS - American College Sports medicine. Progression Models in Resistance Training for Healthy Adults: Position Stand, 2009;687-708.

13. Eiras SB; Silva WH; Souza DL; Vendruscolo R. Factores de adhesión y el mantenimiento de la actividad física por mayor. Rev. Bras. Cienc. Esporte. $2010 ; 31(2): 75-89$.

14. Kearney PM; Whelton M; Reynolds K; Muntner P; Whelton PK; He J. Global burden of hypertension: analysis of worldwide data. Lancet. 2005;365(9455):217-223.

15. Costa JBY; Gerage AL; Gonçalves CGS; Pina FLC; Polito MD. Influencia del estado de entrenamiento en el comportamiento de la presión arterial después de una sesión de entrenamiento con pesas en ancianos hipertensos. Rev Bras Med Esporte. 2010;16(2):103-106.

16. Mediano MFF; Paravidino V; Simão R; Pontes FL; Polito MD. Comportamiento de la presión arterial subaguda después de un entrenamiento de fuerza en hipertensos controlados. Rev Bras Med Esporte. 2005;11(6):337-340.

17. Rondon MUPB; Alves MJ; Braga AM; Teixeira OT; Barretto AC; Krieger EM. e Negrão, C. E. Post-exercise blood pressure reduction in elderly hypertensive patients. J Am Coll Cardiol. 2002;39:676-82.

18. Anunciação, P.G., Polito, M.D. A review on post-exercise hypotension in hypertensive individuals. Arq. Bras. Cardiol. 2010;96(5):100-109.

19. Moraes MR; Bacurau RF; Ramalho JD; Reis FC; Casarini DE; Chagas $\mathrm{JR}$. et al. Increase in kinins on post-exercise hypotension in normotensive and hypertensive volunteers. Biol Chem. 2007;388(5): 533-540.

20. Boroujerdi SS; Rahimi R; Noori SR. Effect of high- versus low-intensity resistance training on postexercise hypotension in male athletes. International SportMed Journal. 2009;10 (2); 95-100.

21. Casonatto J; Polito MD. La hipotensión post-ejercicio aeróbico: una revisión sistemática. Rev Bras Med Esporte. 2009;15(2):151-157. 
22. Brownley KA; Hinderliter AL; West SG; Girdler SS; Sherwood A; Light KC. Sympathoadrenergic mechanisms in reduced hemodynamic stress responses after exercise. Med Sci Sports Exerc. 2003;35(6): 978-86.

23. Floras JS; Sinkey CA; Aylward PE; Seals DR; Thoren PN; Mark AL. Post-exercise hypotension and sympathoinhibition in borderline hypertensive men. Hypertension. 1989;14:28-35.

24. Halliwill JR. Mechanisms and clinical implications of post-exercise hypotension in humans. Exerc Sport Sci Rev. 2001;29(2): 65-70.

25. Legramante JM; Galante A; Massaro M; Attanasio A; Raimondi G; Pigozzi F. et al. Hemodynamic and autonomic correlates of postexercise hypotension in patients with mild hypertension. Am J Physiol Regul Integr Comp Physiol. 2002;282:R1037-43.

26. Goto C; Higashi Y; Kimura M; Noma K; Hara K; Nakagawa K. et al. Effect of different intensities of exercise on endothelium-dependent vasodilation in humans: role of endothelium-dependent nitric oxide and oxidative stress. Circulation. 2003;108:530-5.

27. Halliwill JR; Minson CT.; Joyner MJ. Effect of systemic nitric oxi- de synthase inhibition on postexercise hypotension in humans. J Appl Physiol. 2000;89:1830-6.

28. Lockwood JM; Pricher MP; Wilkins BW; Holowatz LA; Halliwill JR. Postexercise hypotension is not explained by a prostaglandin-dependent peripheral vasodilation. J Appl Physiol. 2005;98:447-53.

29. Cleroux J; Kouame N; Nadeau A; Coulombe D; Lacourciere Y. Aftereffects of exercise on regional and systemic hemodynamics in hypertension. Hypertension. 1992;19:183-91.

30. MacDonald JR; MacDougall JD; Hogben CD. The effects of exercising muscle mass on post exercise hypotension. J Hum Hypertens. 2000;14:317-20.

31. Lizardo JHF; Simões HG. Efeitos de diferentes sessões de exercícios resistidos sobre a hipotensão pós-exercício. Rev Bras Fisioter. 2005;9:289-95

32. Taaffe DR; Galvão DA; Sharman JE; Coombes JS. Reduced central blood pressure in older adults following progressive resistance training. J Hum Hypertens. 2007;21:96-8.

\section{Marco Aurélio Gomes De Oliveira}

oliveira_marcoaurelio@hotmail.com

Universidade do Estado do Pará (UEPA) campus XIII de Tucuruí - Rua 4, nº 20, Santa Mônica. Tucuruí-PA, CEP 68458-100 Fone/Fax, email: (94) 3787-1494.

\section{RECIBIDO: 18-04-2012}

ACEPTADO: 30-05-2012 